\title{
Formation of dislocation loops during He clustering in bcc Fe
}

\author{
N Gao ${ }^{1,3}$, H Van Swygenhoven ${ }^{2,3}$, M Victoria ${ }^{4,5}$ and J Chen ${ }^{1}$ \\ ${ }^{1}$ NES-High Temperature Materials, Paul Scherrer Institute, CH-5232 Villigen PSI, Switzerland \\ 2 NUM/ASQ_-Materials Science and Simulation, Paul Scherrer Institute, CH-5232 Villigen PSI, \\ Switzerland \\ ${ }^{3}$ Ecole Polytechnique Fédérale de Lausanne (EPFL), Institute of Materials (IMX), CH-1015 Lausanne, \\ Switzerland \\ ${ }^{4}$ Lawrence Livermore National Laboratory, PO Box 808 L-370, Livermore, CA 94550, USA \\ ${ }^{5}$ CIEMAT, Madrid, Spain
}

E-mail: helena.vanswygenhoven@psi.ch

Received 2 August 2011

Published 11 October 2011

Online at stacks.iop.org/JPhysCM/23/442201

\begin{abstract}
The clustering of helium in bcc (body centered cubic) iron and the growth of a helium bubble are simulated at the atomistic level for the helium-rich vacancy-poor condition. It is shown that a $\frac{1}{2}\langle 111\rangle$ dislocation loop is formed as a sequential collection of $\langle 111\rangle$ crowdions, the latter being the most stable self-interstitial atom configuration in the presence of a He cluster.

(Some figures in this article are in colour only in the electronic version)
\end{abstract}

Because of their low solubility in the metal matrix, helium atoms will cluster and eventually form helium bubbles when they are introduced in a metal lattice by transmutation during neutron irradiation $(\mathrm{n}, \alpha)$ or via direct $\alpha$-implantation [1, 2]. The resulting defects degrade the material's properties and thus play an important role in the mechanical integrity of the metals when used for structural components in fusion and fission reactors [1]. The modification of the microstructures due to irradiation can be studied largely by computer simulations involving all length scales with the aim of predicting the lifetime behavior of the irradiated materials. At the microscopic level, understanding the clustering mechanism of helium atoms, the formation of helium-vacancy clusters $\left(\mathrm{He}_{n} \mathrm{~V}_{m}\right)$ and the growth of such a cluster into a bubble is a prerequisite for simulating more complex phenomena at longer time scales and larger helium doses. Since there are no experimental tools available for studying the initial clustering of helium to a nanometer sized bubble, the kinetics of these processes is usually addressed via atomistic simulations. By calculation the energy of binding of helium atoms to $\mathrm{He}_{n} \mathrm{~V}_{m}$ and $\mathrm{He}_{n-1} \mathrm{~V}_{2}$ clusters with DFT or molecular dynamics (MD) methods [3-6], an estimation of the number of helium atoms that can reside in a cluster can be obtained. The further growth of the cluster into a bubble with increasing helium content is addressed by using molecular dynamics or Monte Carlo (MC) techniques because of the increasing number of particles and time scale involved [7-10].

For the special condition of continuous helium but insufficient vacancy supply, the growth of bubbles in metals has been analytically described as a pressure-driven athermal process, where the pressure is high enough to punch out an interstitial dislocation loop the size of the bubble radius [11, 12]. The process is schematically presented in figure 1 . Building further on that theory, it has been suggested that the loop might be formed by an accumulation of single self-interstitials emitted separately from the bubble [13]. The presence of loops in the neighborhood of helium bubbles has been experimentally observed using TEM [14, 15]. Dislocation loops are however also observed as a result of heavy ion irradiation without the presence of helium. For iron and iron-based ferritic/martensitic steels [16, 17], two sets of interstitial dislocation loops have been found, with Burgers vectors of $\frac{1}{2} a_{0}\langle 111\rangle$ and $a_{0}\langle 100\rangle$, respectively. Although loop punching is accepted as a viable mechanism for 


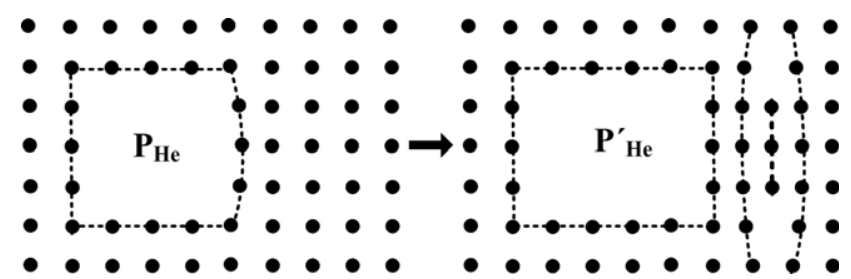

Figure 1. The schematic map adapted from [11] for the loop punching mechanism described by the pressure-driven model.

bubble growth, the kinetic formation process has never been experimentally observed in situ or simulated at the atomistic level.

In this paper the clustering of helium in bcc iron and the growth of a helium bubble are simulated at the atomistic level for the helium-rich vacancy-poor situation which represents a low temperature irradiation condition or a sub-threshold irradiation in which there is only the thermal vacancy concentration available. It is shown that a $\frac{1}{2}\langle 111\rangle$ dislocation loop is formed when an increasing number of helium atoms are absorbed in a helium-vacancy cluster. In contrast to the pressure-driven loop punching mechanism case, the loop is formed by sequential emission of $\langle 111\rangle$ crowdions or $\langle 110\rangle$ dumbbells with increasing helium accumulation, finally aligning up as crowdions to form a prismatic dislocation loop configuration that can move away from the bubble. Such a sequential process keeps the $\mathrm{He} / \mathrm{V}$ ratio below 1.75 .

The simulations have been performed with a magnetic $\mathrm{Fe}-\mathrm{Fe}$ potential [18]. These potentials correctly represent the energetics of the different defect configurations, in agreement with DFT calculations [19]. The $\langle 110\rangle$ interstitial dumbbell is the lowest energy configuration in magnetic $\mathrm{Fe}$, which is different from the case for other bcc metals where the $\langle 111\rangle$ crowdion is found to be the most stable configuration [20]. The $\mathrm{Fe}-\mathrm{He}$ interaction is described through a pair potential as detailed in [21], also used to study the energy of binding of the self-interstitial $\mathrm{Fe}$ and $\mathrm{He}$ atoms to a $\mathrm{He}_{n} \mathrm{~V}$ cluster [4]. A computational box of 50 unit cells is relaxed using the Parrinello-Rahman [22] algorithm with a 0.1 fs time step at $300 \mathrm{~K}$. Such a small time step is necessary for simulating the lightweight helium atoms. Helium atoms are inserted one by one in a configuration that is quenched to $0 \mathrm{~K}$ with molecular statics (MS). After each helium atom insertion, $60000 \mathrm{MS}$ steps are performed, followed by a temperature rescaling to $300 \mathrm{~K}$ and an additional $100000 \mathrm{MD}$ steps to allow the defect configuration to relax at $300 \mathrm{~K}$. For the insertion of the next helium atom, the configuration is quenched back to $0 \mathrm{~K}$ and a similar procedure is repeated.

The first helium atom is inserted in a tetrahedral interstitial site which, according to DFT calculations, is energetically the most favorable interstitial position for a helium atom [3]. On gradually inserting more helium atoms up to 5, the maximal displacement of the $\mathrm{Fe}$ atoms around the helium cluster increases but stays below $1 \AA$, as shown in figure 2. The insertion of the sixth He results in a displacement of $3 \AA$ of one of the Fe atoms which is observed after 4500 MD steps. The defect appears as a [111] crowdion.

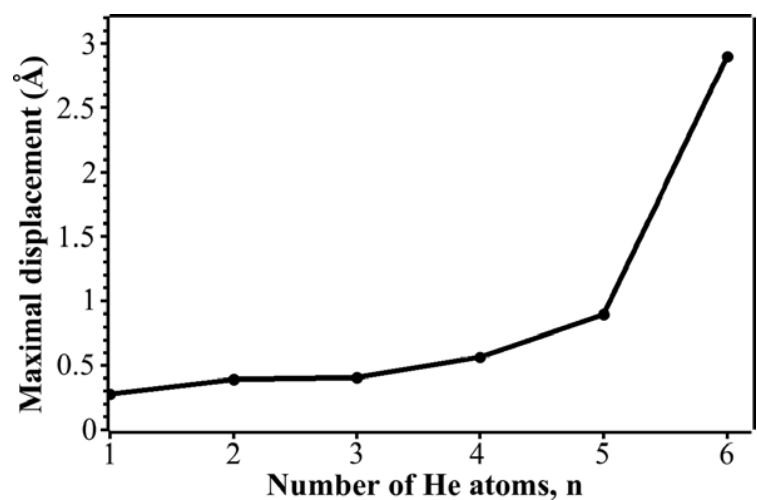

Figure 2. The maximum displacement of $\mathrm{Fe}$ atoms surrounding a small He cluster up to $n=6$.

To explore the stability of the defect, 350000 additional MD steps were carried out during which the defect changes via an intermediate [101] dumbbell configuration into a [111] crowdion. The three observed configurations of the defect are shown in figure 3. It is worth noting that the dumbbell configuration was only observed during 4000 of the 350000 MD steps carried out. Detailed inspection demonstrated that the change in defect configuration is caused by a rearrangement of the six helium atoms in the vacancy-type defect. Several simulations with different initial positionings of the helium atoms have been carried out, demonstrating that no matter where the sixth helium atom is positioned, the first emitted SIA (self-interstitial atom) ends up in a crowdion configuration close to the helium cluster when enough MD steps are carried out.

During the insertion of more helium atoms into the $\mathrm{He}_{6} \mathrm{~V}$ cluster, the average displacement of the surrounding $\mathrm{Fe}$ atoms increases again and after the insertion of 13 helium atoms, a second SIA is emitted in a $\langle 110\rangle$ dumbbell configuration. In the first inset in figure 4 the helium cluster configuration (gray interconnected circles) is shown together with the first [111] crowdion (green) and the second SIA in the form of a [011] dumbbell (red) after $18000 \mathrm{MD}$ steps. Further relaxation of the sample at $300 \mathrm{~K}$ causes a sequential transition from the [011] dumbbell via a [1111] crowdion (after $4000 \mathrm{MD}$ steps) into a [1110] dumbbell (after a further $4000 \mathrm{MD}$ steps), to end finally after $2000 \mathrm{MD}$ steps in a [1 111 crowdion where it remains stable during the remaining $72000 \mathrm{MD}$ steps. The final configuration of the two crowdions is also shown as an inset in figure 4 . The same figure also shows the energy barriers and the energies of all intermediate configurations calculated using the nudged elastic band method [23], demonstrating that the observed sequential configurations are driven by a search for a lower energy state. Note that the [1 $\overline{1} 1]$ and [ $\overline{1} \overline{1} 1]$ crowdions both lie in a (011)-type plane separated by one extra lattice plane.

Insertion of the 14th helium atom followed by relaxation at $300 \mathrm{~K}$ causes the emission of a third crowdion, which is lying on the (110) plane in between the first SIA and the second SIA, having the same direction as the first crowdion, i.e. along the [1-11] direction. The fourth SIA is then emitted as a crowdion after the insertion of 18 helium atoms having 


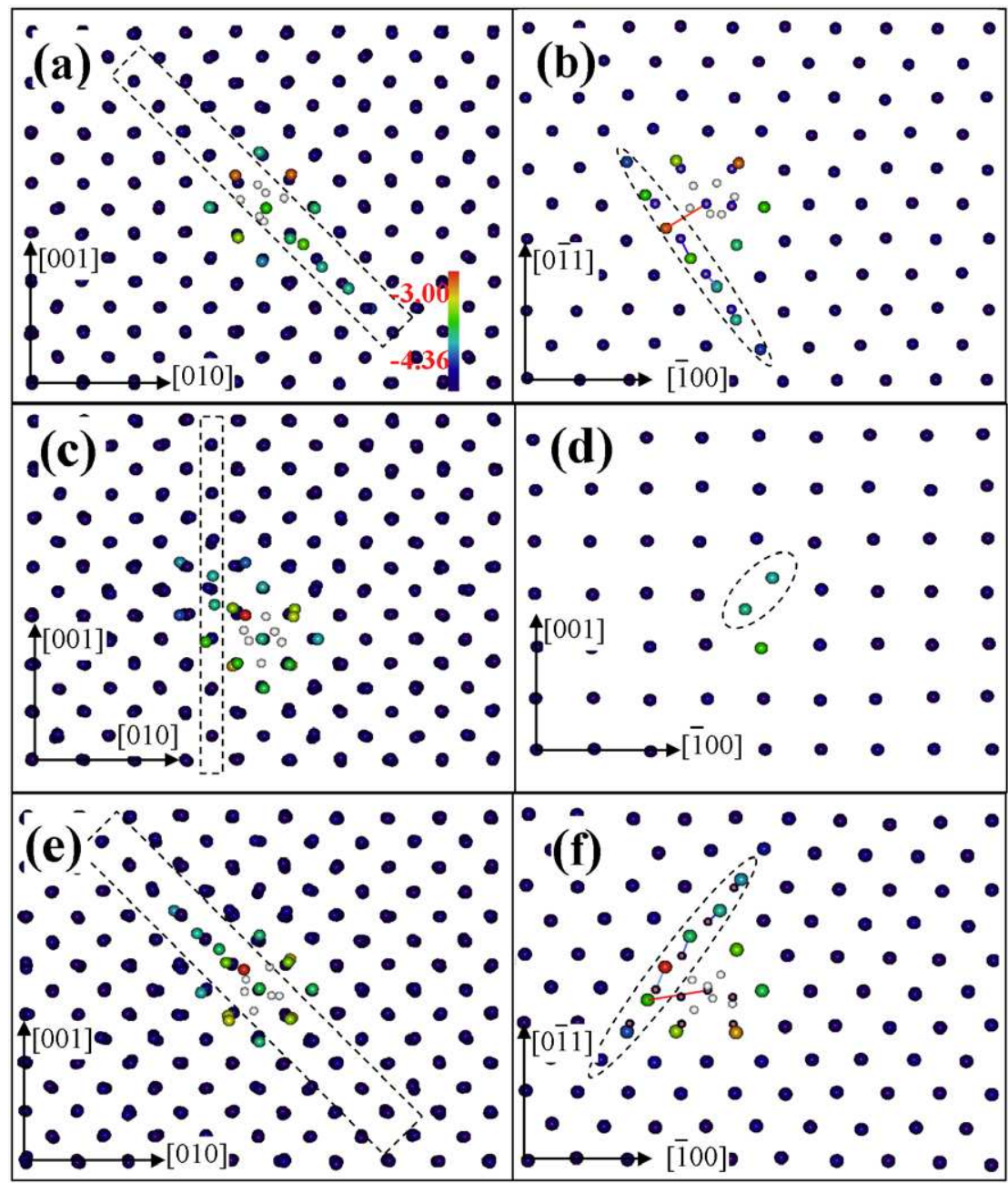

Figure 3. The three states for the formation of the first SIA with six He atoms. The configuration viewed along the [100] direction is shown in (a), (c) and (e). The atomic layer containing the $\langle 111\rangle$ crowdion or $\langle 110\rangle$ dumbbell is shown in (b), (d) and (f). The small gray particles are helium atoms and the color of the Fe atom is identified according to its cohesive energy which has a range from -4.36 to $-3.0 \mathrm{eV}$. The big displacement vectors connecting initial sites (shown by smaller dark particles with points at the center) and relaxed states are included.

the same direction as the second SIA. In other words, the cluster $\mathrm{He}_{18} \mathrm{~V}_{4}$ is surrounded by four crowdions from which two are directed along the [ī1 1$]$ direction and two along the [1111] direction, all lying in adjacent (011) planes.

The fifth SIA and sixth SIA are ejected in the form of dumbbells after respectively 23 and 27 helium atoms have been inserted. They transform into $\langle 111\rangle$ crowdions when the seventh SIA (with 29 helium atoms) is emitted in a [111] crowdionconfiguration. During this process, also the previous emitted SIAs all align into the same [1111] direction as the crowdions. After having inserted 29 helium atoms the seven-SIA crowdion cluster moves a $\frac{1}{2}\langle 111\rangle$ lattice vector, i.e. a Burgers vector, away from the helium bubble. Close inspection of the helium cluster reveals an ellipsoidal shape with the seven-SIA interstitial cluster parallel to the long axis. During further insertion of helium atoms, the SIA cluster is observed to rotate, meanwhile absorbing 11 additional SIAs that are formed as [111] crowdion configurations allowing the ellipsoid to grow spherically. Figure 5 shows the configurations of the growing SIA cluster projected on a (100) plane, and pictures the He cluster surrounded by SIAs when it contains $29 \mathrm{He} \mathrm{(a),} 33 \mathrm{He}$ (b) and $35 \mathrm{He}$ atoms (c). The inset in figure 5(c) shows the SIA cluster configuration of 18 SIAs in the (1 $1 \overline{1} \overline{1})$ habit plane, demonstrating that they are arranging themselves as if they would form part of a hexagonal dislocation loop, the open circles figuring as the empty positions in this loop.

Further insertion of helium atoms results in the ejection of more SIA atoms on all sides of the helium bubble. These SIAs are observed to diffuse and reorient in order to align

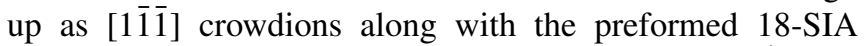
cluster. After insertion of 61 helium atoms, a perfect $\frac{1}{2}\langle 111\rangle$ hexagonal dislocation loop of 24 SIAs is formed. Now all open circles in the inset of figure 5(c) are filled with SIA Fe atoms. Figure 5(d) shows the perfect dislocation loop viewed along the [110] direction, traveling 8-9 Burgers vectors away from the helium bubble after 58000 MD steps.

The above atomistic simulations demonstrate that a $\frac{1}{2}\langle 111\rangle$ interstitial dislocation loop is formed when He clusters into a bubble in a vacancy-poor environment. The dislocation loop forms from a collection of aligning $\langle 111\rangle$ crowdions. In contrast to what is observed for the stability of SIA defect 


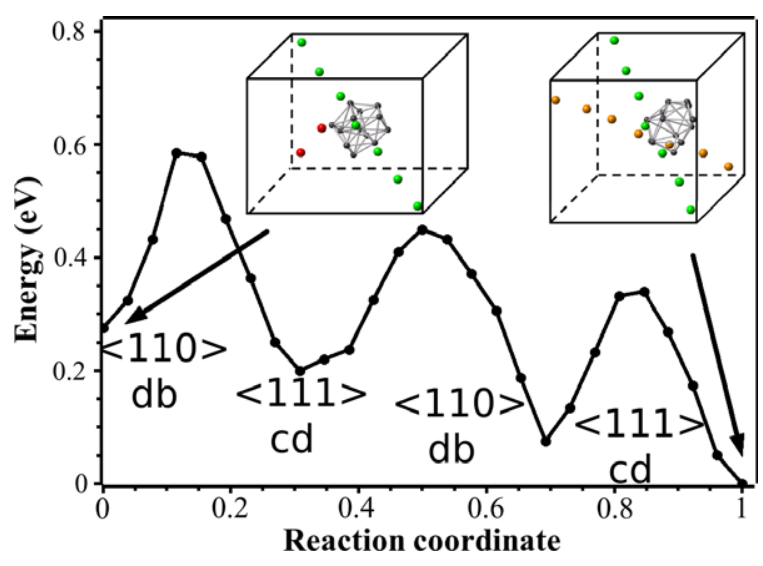

Figure 4. The energies of migration between the four observed configurations of second SIA emitted after 13 helium atoms ( $\mathrm{db}$ and cd stand for dumbbell and crowdion, respectively). The two configurations shown are the configuration with $13 \mathrm{He}$ atoms (gray interconnected atoms) with (left) the initial crowdion

(green)-dumbbell (red) configuration and (right) the final two dumbbells with different directions.

configurations in the perfect Fe lattice, the $\langle 111\rangle$ crowdion turns out to be the stable defect configuration in the presence an over-pressurized helium cluster. The simulations show that after emission of seven SIAs, all interstitials align along the same [11ㅣ direction to form an SIA cluster. During further supply of helium atoms, the small defect cluster is still able to move around the helium bubble looking for the $\langle 111\rangle$-type orientation with the lowest constraints, meanwhile absorbing all additional ejected SIA atoms. In this way, a $\frac{1}{2}\langle 111\rangle$ hexagonal dislocation loop is formed that can move away from the helium bubble. It is to be expected that the intermediate configurations of the SIAs before they align up with the already existing crowdion cluster will depend on the simulation time and the initial positioning of the helium atoms in the cluster. To check the influence of the empirical potential, similar simulations have been performed using the Ackland Fe-Fe potential [24], the Juslin-Nordlund $\mathrm{Fe}-\mathrm{He}$ potential [25] and the Beck He-He potential [26]. The mechanism observed for the clustering of SIAs into a dislocation loop was very similar but the numbers differ: a prismatic loop was only observed after emission of 44 SIAs corresponding to a bubble containing $110 \mathrm{He}$ atoms.

To check the role of the He pressure during the bubble growth, the $\mathrm{He} / \mathrm{V}$ ratio was calculated. The $\mathrm{He} / \mathrm{V}$ ratio was obtained as the ratio of the average volume of a helium atom in the helium cluster (calculated using the Voronoi algorithm implemented in the code voro ${ }^{++}$[27]) to the volume of the Fe vacancy. As shown in figure 6, on average the $\mathrm{He} / \mathrm{V}$ remains below 1.75 with dip values of the order of 1.6. Such low values are observed for the emission of the first SIA and the configuration obtained after $35 \mathrm{He}$ atoms, corresponding to the diffusion of the seven-SIA cluster and the rapid collection of 11 additional SIAs. Also the movement of the seven-SIA cluster to a Burgers vector away from the cluster $(29 \mathrm{He}$

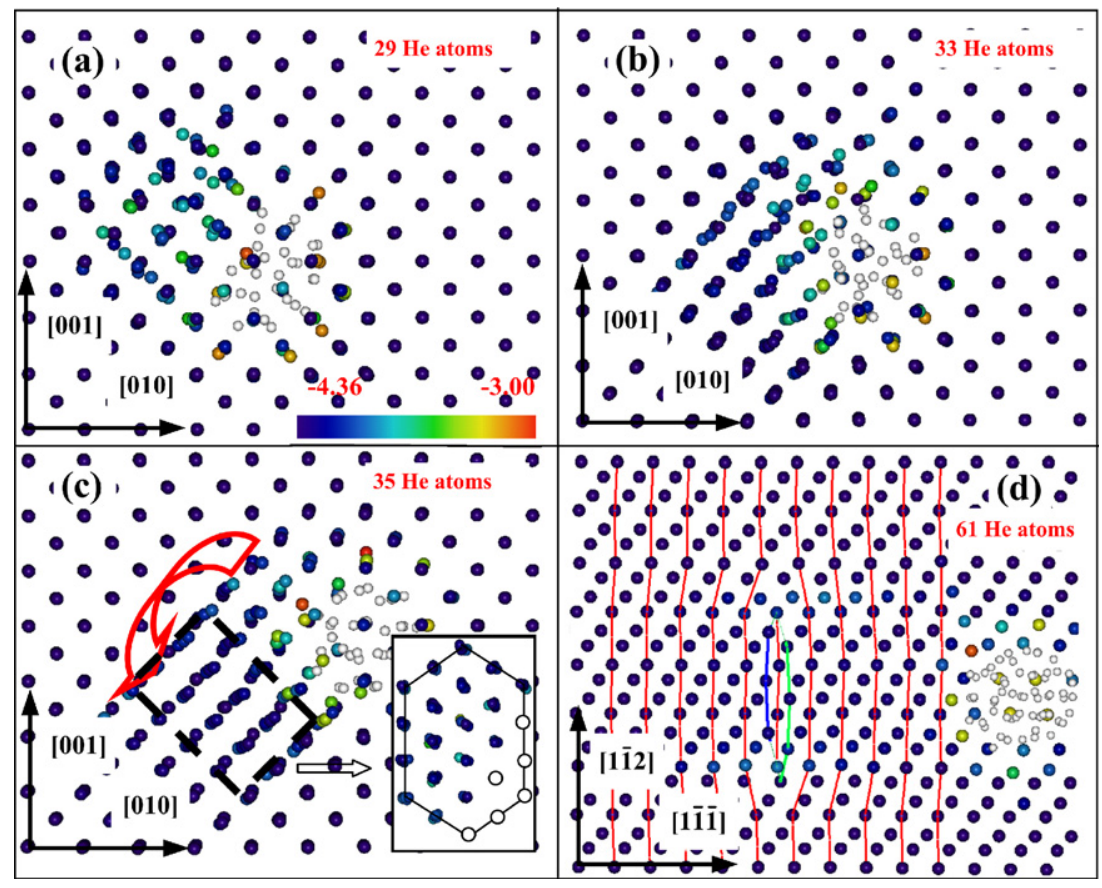

Figure 5. Configurations of the growing SIA cluster projected on the (100) plane together with the He cluster (open circles): (a)-(c) include 29,33 and $35 \mathrm{He}$ atoms respectively. The shape of loop is shown in the inset image in (c) where open circles indicate SIA positions that are still missing for forming 24 hexagonal SIAs loop. The Fe atoms are colored according to the cohesive energy from -4.36 to $-3.0 \mathrm{eV}$ as shown by the color map. The 24-SIA dislocation loop traveling away from the 61-helium cluster is shown in (d) where the B layers of the $\mathrm{ABC}$ sequence along the $\langle 111\rangle$ direction (viewed along the [110] direction) are shown with red dashed lines. The $\mathrm{A}$ and $\mathrm{C}$ layers of the loop are shown with blue and green lines. 


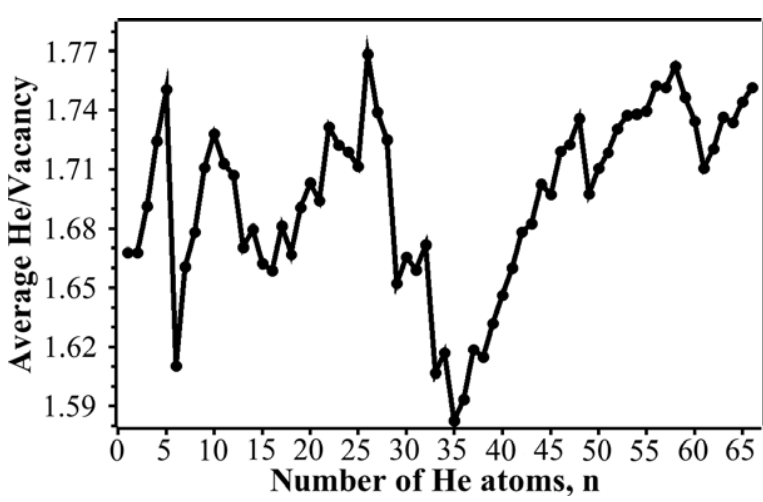

Figure 6. The average helium to vacancy ratio with increasing number of helium atoms.

atoms) is marked by a dip in the $\mathrm{He} / \mathrm{V}$ ratio, demonstrating the stress relief during this procedure.

In summary, molecular dynamics simulations demonstrate that loops can be formed during bubble growth under poor vacancy supply, but the mechanism at atomic level by which the dislocation loop is formed is different from the analytical pressure induced punching mechanism. The simulations show that $\frac{1}{2}\langle 111\rangle$ dislocation loops are formed in Fe, but as a sequential collection of $\langle 111\rangle$ crowdions, the latter being the most stable SIA configuration in the presence of a He cluster.

This work was performed within the Swiss Generation IV Program. It was partially financed by the EU-project GetMat.

\section{References}

[1] Ullmaier H and Schilling W 1980 Physics of Modern Materials Vol. 1 (Vienna: International Atomic Energy Agency)

[2] Donnelly S E 1985 Radiat. Effects 901

[3] Fu C C and Willaime F 2007 J. Nucl. Mater. 367-370 244
[4] Gao N, Victoria M, Chen J and Van Swygenhoven H 2011 J. Phys.: Condens. Matter 23245403

[5] Stewart D M, Osetsky Yu N, Stoller R E, Golubov S I, Seletskaie T and Kamenski P J 2010 Phil. Mag. 90935

[6] Hafez Haghighat S M, Lucas G and Schäublin R 2009 Europhys. Lett. 8560008

[7] Yang L, Zu X T, Xiao H Y, Gao F, Heinisch H L, Kurtz R J and Liu K Z 2006 Appl. Phys. Lett. 88091915

[8] Caro A, Hetherly J, Stukowski A, Caro M, Martinez E, Srivilliputhur S, Zepeda-Ruiz L and Nastasi M 2011 J. Nucl. Mater. 418261

[9] Morishita K 2007 Phil. Mag. 871139

[10] Deo C S, Okuniewski M A, Srivilliputhur S G, Maloy S A, Baskes M I, James M R and Stubbines J F 2007 J. Nucl. Mater. 361141

[11] Evans J H 1978 J. Nucl. Mater. 76/77 228

[12] Greenwood G W, Foreman A J E and Rimmer D E 1959 J. Nucl. Mater. 4305

[13] Trinkaus H and Wolfer W G 1984 J. Nucl. Mater. 122/123 552

[14] Evans J H, van Veen A and Caspers L M 1981 Nature 291310

[15] Chen J, Jung P, Hoffelner W and Ullmaier H 2008 Acta Mater. 56250

Chen J, Jung P and Trinkaus H 1999 Phys. Rev. Lett. 822709

[16] Xu S, Yao Z and Jenkins M L 2009 J. Nucl. Mater. 386-388 161

[17] Zinkle S J and Singh B N 2006 J. Nucl. Mater. 351269

[18] Chiesa S, Derlet P M and Dudarev S L 2009 Phys. Rev. B 79214109

Dudarev S L and Derlet P M 2005 J. Phys.: Condens. Matter 177097

[19] Fu C C, Willaime F and Ordejon P 2004 Phys. Rev. Lett. 92175503

[20] Nguyen-Manh D, Horsefield A P and Dudarev S L 2006 Phys. Rev. B 73 020101(R)

[21] Gao N, Samaras M and Van Swygenhoven H 2010 J. Nucl. Mater. 400240

[22] Parrinello M and Rahman A 1981 J. Appl. Phys. 527182

[23] Henkelman G, Uberuaga B P and Jónsson H 2000 J. Chem. Phys. 1139901

[24] Ackland G J, Mendelev M I, Srolovitz D J, Han S and Barashev A V 2004 J. Phys.: Condens. Matter 16 S2629

[25] Juslin N and Nordlund K 2008 J. Nucl. Mater. 382143

[26] Beck D E 1968 Mol. Phys. 14311

[27] Voro $^{++}$software library http://math.lbl.gov/voro++ 